\title{
Gênero, segurança alimentar e nutricional e vulnerabilidade: o Programa das Mulheres Mil em foco
}

\author{
Gender, food and nutrition security and vulnerability: \\ the Thousand Women Program in focus
}

Jussara Maysa Campos (https://orcid.org/0000-0002-8837-8475) ${ }^{1}$

Rita de Cássia Coelho de Almeida Akutsu (https://orcid.org/0000-0003-0699-7617) ${ }^{1}$

Izabel Cristina Rodrigues Silva (https://orcid.org/0000-0002-6836-3583) ${ }^{2}$

Karin Savio Oliveira (https://orcid.org/0000-0002-2848-0427) ${ }^{2}$

Renata Monteiro (https://orcid.org/0000-0001-9188-235X) ${ }^{2}$

${ }^{1}$ Departamento de Nutrição, Universidade de Brasília. Campus Universitário Darcy Ribeiro s/n, Asa Norte. 70910-900 Brasília DF Brasil.

jussara.maysa@gmail.com

${ }^{2}$ Farmácia, Universidade de Brasília. Brasília DF Brasil.

\begin{abstract}
Gender inequality is revealed in the issues of education, income and access to Food and Nutrition Security. In Brazil, the National Women Thousand Program aims to intensify the process of regional and institutional development by improving the access of women in situations of social vulnerability to education and the world of work. The objective is to analyze the contribution of the Programa Nacional Mulheres Mil's to reduce vulnerability to food insecurity of households headed by woman. A semi-structured questionnaire was applied to 384 participants, which contained the demographic and socioeconomic variables as well as the Brazilian Food Insecurity Scale. It was observed that, although there was no change in the Food Security situation, there were significant differences in relation to income, especially among women in situation of food insecurity, varying from $20.5 \%$ to $46 \%$ the number of participants with an income range higher than one minimum wage. The advance in the identified income was not enough to intervene in Food Insecurity. Further research should be carried out in order to deepen the understanding of the relationships between income, education and food and nutritional security, especially from the point of view of actions directed at the female gender. Key words Food Security and Nutrition, Social Vulnerability and Education
\end{abstract}

Resumo A desigualdade de gênero se revela nas questões de educação, renda e acesso a Segurança Alimentar e Nutricional. No Brasil, o Programa Nacional Mulheres Mil tem como objetivo intensificar o processo de desenvolvimento regional $e$ institucional, pela melhoria do acesso de mulheres em situação de vulnerabilidade social à educação e ao mundo do trabalho. O objetivo é analisar a contribuição do Programa para redução da vulnerabilidade à insegurança alimentar dos domicílios chefiado por mulheres. Aplicou-se um questionário semiestruturado para 384 participantes contendo as variáveis demográficas e socioeconômicas e a Escala Brasileira de Insegurança Alimentar. Constataram-se diferenças significativas em relação à renda, especialmente, entre aquelas em situação de insegurança alimentar, variando o número de participantes da faixa de renda superior a um salário mínimo. $O$ avanço na renda não foi suficiente para intervir na Insegurança Alimentar. Mais pesquisas devem ser realizadas com objetivo de aprofundar a compreensão das relações entre renda, educação e Segurança Alimentar e Nutricional, sobretudo sob a ótica das ações direcionadas ao gênero feminino.

Palavras-chave Segurança Alimentar e Nutricional, Vulnerabilidade Social e Educação 


\section{Introdução}

Diversos autores em estudos que já se tornaram clássicos apontam as relações entre o ato de alimentar-se e o alimento permeado pelo gênero ${ }^{1}$. Nos dias atuais, essas relações se expressam na alta prevalência de insegurança alimentar ligada, além dos fatores nutricionais e de estilo de vidas, ao nível socioeconômico e ao gênero do chefe da família ${ }^{2}$. A desigualdade de gênero se revela nas questões de segurança alimentar e nutricional (SAN) como um dos resultantes da desigualdade do acesso e controle dos recursos financeiros ${ }^{3,4}$. Adicionalmente, ressalta-se que o acesso à educação é um dos indicadores de discriminação de gênero ${ }^{5} \mathrm{e}$, consequentemente, da maior vulnerabilidade à Insegurança Alimentar.

Considerando a importância dos recursos produtivos para a determinação da situação de pobreza e de insegurança alimentar, Siliprandi ${ }^{6}$ ressalta a fácil compreensão da maior vulnerabilidade de crianças e mulheres em relação à fome e desnutrição. Tal vulnerabilidade se deve a um aspecto específico das relações de gênero e da segurança alimentar que é a imbricação entre a identidade de gênero e a invisibilidade do trabalho das mulheres no campo da alimentação ${ }^{7}$ e no modo como a economia capitalista se assenta sobre o trabalho não pago realizado pelas mulheres.

A dificuldade de acesso aos alimentos, por um contingente significativo de nossa população, sempre foi considerada a barreira principal para a segurança alimentar e nutricional no Brasil. Embora a condição de segurança alimentar abranja muitos outros aspectos, é fato que a falta de renda para a aquisição dos alimentos adequados em quantidade suficiente mostra-se como o fator principal da insegurança alimentar ${ }^{8}$.

Maluf $^{3}$ ao discorrer sobre o nível de renda e a insegurança alimentar destaca que os preços dos alimentos representam um importante determinante do poder de compra dos salários e demais rendas oriundas do trabalho dos cidadãos, sendo os segmentos de menor renda os mais afetados pelas variações de preço desses produtos. Nesses segmentos, os gastos com a alimentação podem comprometer uma parcela significativa da renda familiar, expondo-os, portanto, à insegurança alimentar ${ }^{8,9}$.

No Brasil o número de mulheres que chefiam domicílios passou de $22,2 \%$ para $38,8 \%$ entre 2000 e $2013^{4}$ chegando a 57,3 milhões de domicílios sob o comando feminino. De acordo com o $\mathrm{IBGE}^{10}$, o rendimento médio real mensal de todas as fontes, das mulheres de 10 anos ou mais de idade correspondia a $65 \%$ da renda dos homens. Em 2010, subiu para $68 \%{ }^{10}$, o que revela o avanço das mulheres, mas ainda longe de alcançar a igualdade.

Infelizmente, os resultados da Pesquisa Nacional de Amostra por Domicílios ${ }^{8}$, indicaram que ainda persiste no país a maior vulnerabilidade à insegurança alimentar dessas famílias que a pessoa de referência é do sexo feminino ${ }^{8}$.

Como bem destacam Brito e Costa ${ }^{2}$, no Brasil, a segurança alimentar, à semelhança de outras condições sociais apresenta uma relação de gênero, que influencia negativamente as chances de existência de insegurança alimentar moderada nos domicílios chefiados por mulheres, quando comparados aos chefiados por homens. Além disso, como mostrado nos estudos citados ${ }^{11}$, as mulheres carecem de ações que as tornem autônomas e empoderadas na medida em que seu papel como protetora da família está posto.

Considerando o papel crucial das mulheres enquanto vínculo do plano biológico, social e econômico em especial na SAN doméstica, Le Bihan et al. ${ }^{12}$ e Maluf ${ }^{3}$ destacam a relevância de ações voltadas para a condição feminina nos aspectos educacionais e profissionais, entre outros ${ }^{2}$.

No período de 2007 a 2010, iniciou-se no Brasil o "Projeto Mulheres Mil" que visava à formação educacional, profissional e cidadã de mulheres em vulnerabilidade social e que residiam nas regiões norte e nordeste. Foi concebido a partir de uma cooperação internacional com os colleges comunitários canadenses e os Centros Federais de Educação Tecnológica (CEFETs) das regiões mencionadas.

Em 2011, o Ministério da Educação instituiu nacionalmente o Programa Mulheres Mil, em substituição ao Projeto inicial, como uma das ações do Plano Brasil Sem Miséria, tendo como principais diretrizes: 1) o acesso à educação; 2) a redução de desigualdades sociais e econômicas de mulheres; 3) a inclusão social; 4) a igualdade de gênero e 5) combate à violência contra a mulher. Para tanto, desenvolveu metodologia própria de Acesso, Permanência e Êxito ${ }^{13}$.

O programa também integrou as ações previstas no "III Plano de Políticas para as Mulheres" (2013-2015), na temática igualdade de gênero e autonomia econômica, compondo a linha da ação que tratada da inserção e permanência das mulheres em relações formais de trabalho não discriminatórias em razão de sexo, origem, raça, etnia, classe social, idade, orientação sexual, identidade de gênero ou deficiência, com igualdade de rendimentos e fomento à ascensão social e à permanência em cargos de direção. 
De acordo com os documentos oficiais a metodologia de Acesso, Permanência e Êxito propõe para além de uma qualificação profissional, uma formação cidadã pautada na transversalidade de temas, tais como: saúde, direitos da mulher, empreendedorismo e autoestima, entre outros ${ }^{14}$.

Diante da maior vulnerabilidade à insegurança alimentar dos domicílios chefiados por mulheres, bem como dos aspectos socioeconômicos relacionados a esta condição, em especial, renda e educação, o presente trabalho teve por objetivo analisar como o Programa Nacional Mulheres Mil pode contribuir para redução da vulnerabilidade à Insegurança Alimentar.

\section{Métodos}

Trata-se de um estudo descritivo, longitudinal, prospectivo de intervenção, que investigou participantes do Programa Mulheres Mil, nas cinco regiões geográficas, em 11 estados brasileiros e no Distrito Federal, representados por: Boa Vista-RR; Palmas e Araguatins-TO; Belém-PA; Jacobina-BA; Quixelô e Iguatu-CE; Maceió, Satuba e Arapiraca-AL; Inhumas e Ceres-GO; Taguatinga-DF; Cabedelo-PB; Vitória-ES; Realengo-RJ; Lages, Videira e Florianópolis-SC.

Utilizou-se uma amostra semi-probabilística de 384 participantes, selecionadas a partir de três estágios (1: análise das unidades ofertantes do Programa Mulheres Mil em 2013; 2: sorteio dos Institutos Federais de Educação Profissional e Tecnológica (IFs) ofertantes; 3: adesão dos IFs e campi que ofertaram o programa no período de 2013 a 2014). Considerou-se a distribuição normal para o critério de cálculo de tamanho amostral, com erro amostral de $8 \%$, nível de confiança de $95 \%$ e a distribuição de resposta de 50\%. No primeiro estágio, realizou-se a divulgação da pesquisa e o convite para os 37 IFs que executavam o programa, tendo como parâmetro de participação regional a divisão proporcional de campi da Rede Federal em nível nacional, de acordo com o Sistema de Informação da Educação Profissional - SISTEC.

Anteriormente à aplicação dos questionários, realizou-se a validação dos instrumentos, realização do projeto piloto para treinamento e ajustes da estratégia de campo.

A coleta de dados foi realizada ao início do curso (até dois meses) e após, ao menos, dois meses da conclusão do curso. A primeira etapa foi aplicada pelos gestores do programa após o recebimento de orientações da pesquisa e, a se- gunda etapa junto às egressas foi realizada, por entrevista, via telefone, por equipe devidamente treinada. As duas etapas de coleta de dados foram realizadas a partir do segundo semestre de 2013 e durou até o final de 2014, obedecendo aos calendários acadêmicos das instituições envolvidas na pesquisa.

A seguir são apresentadas detalhadamente cada uma das dimensões avaliadas durante a coleta de dados.

\section{Variáveis demográficas e socioeconômicas}

Considerando a relação entre a insegurança alimentar e as condições socioeconômicas, para caracterização dos domicílios das participantes da pesquisa, selecionaram-se as seguintes variáveis demográficas socioeconômicas:

1 - localização da residência (zona urbana/ área rural), 2 - faixa etária, 3 - estado civil, 4 escolaridade, 5 - renda mensal, 6 - número de dependentes, 7 - identificação da principal fonte de renda do domicílio e, 8 - gastos da família com alimentação.

As variáveis foram determinadas em função de sua relação com a situação de segurança alimentar ${ }^{3,10,15}$

\section{Situação de Insegurança Alimentar}

Utilizou-se para o diagnóstico de insegurança alimentar, a "Escala Brasileira de Insegurança Alimentar domiciliar - EBIA", instrumento validado no país, no período de 2003 a 2004, a partir do trabalho integrado de pesquisadores de cinco universidades brasileiras ${ }^{16}$. Durante o processo de validação da versão brasileira, observou-se tanto na população urbana quanto na rural um Alfa de Cronbach variando entre 0,87 a 0,95 , demonstrando alta validade interna do instrumento.

Após a utilização da EBIA em vários estudos foi proposta a supressão de uma pergunta e após testes estatísticos (Análise de Rasch), a escala foi validada com 14 itens, e posteriormente utilizada em estudo com 1.637 usuários do Programa Restaurantes Populares ${ }^{17}$.

Nesta pesquisa aplicou-se a versão do instrumento com 14 itens. A aplicação nesta pesquisa foi realizada em até dois meses após o início das aulas e, no mínimo, após dois meses depois da conclusão do curso. Na presente pesquisa o instrumento obteve um Alpha de Cronbach de 0,90, considerado excelente.

Em virtude de a presente pesquisa abordar a associação da condição da segurança/inseguran- 
ça alimentar enquanto característica de vulnerabilidade social e seus determinantes sociais (escolaridade, renda etc.), optou-se por considerar a presença ou ausência de Insegurança Alimentar desconsiderando os diferentes níveis (leve, moderada ou grave). Nesse sentido, uma resposta positiva a EBIA foi considerada suficiente para a observação da Insegurança Alimentar do domicílio. Cabe destacar que Carter et al..$^{18}$ ao mensurar a segurança alimentar na Nova Zelândia, optou pela mesma conduta ao aplicar a escala própria de segurança alimentar no sentido de considerar como inseguro a indicação de uma resposta positiva a qualquer um dos questionamentos.

\section{Análise de dados}

Quanto à análise dos dados, foram verificados os requisitos necessários para minimizar a possibilidade de erros nas decisões estatísticas, sendo realizadas conferências sistemáticas entre os dados lançados e os dados coletados nos instrumentos originais da pesquisa. Esses dados foram introduzidos em um banco específico para esta pesquisa, elaborado no Programa Statistical Package for Science - SPSS ${ }^{\oplus}$ na versão 22.0, no qual também foram processadas as análises. Após a criação do formulário de entrada dos dados, foi realizada a checagem dos mesmos por meio da análise de distribuição de frequência, comparando-se os valores de cada variável no banco de dados do SPSS ${ }^{\circledast}$ com aqueles possíveis de ocorrência, evitando-se a ocorrência de erros na digitação.

Posteriormente à caracterização dessas variáveis realizaram-se cruzamentos entre os resultados e o estado de (in)segurança alimentar domiciliar das entrevistadas com objetivo de identificar as prováveis relações existentes nos diferentes grupos (com Segurança Alimentar - SA ou com Insegurança Alimentar - IA).

Procedeu-se com a descrição dos dados em termos de frequências relativas e medidas resumo como a média e o desvio padrão. Para as análises de associação, o teste estatístico utilizado foi o qui-quadrado, e para a diferença de médias, os pressupostos da normalidade foram checados, via teste de Shapiro-Wilk. Os testes estatísticos utilizados foram t de Student para comparar os dois momentos de avaliação e Kruskal-Wallis para apurar a análise de variância, tendo Bonferroni como Post-hoc. O nível de significância adotado foi de $5 \%$. A presente pesquisa foi aprovada pelo Comitê de Ética da Faculdade de Ciências da Saúde da Universidade de Brasília (UnB).

\section{Resultados}

\section{Variáveis Demográficas e Socioeconômicas}

Considerando as variáveis demográficas e socioeconômicas pesquisadas, os resultados demonstraram que Renda e Número de Pessoas do Domicílio foram as variáveis que apresentaram diferença estatística nos diferentes momentos investigados, ou seja, no Início e Após a Conclusão do curso. O detalhamento está apresentado na Tabela 1.

\section{Situação de Insegurança Alimentar}

Quanto à situação de Insegurança Alimentar, verificou-se que a participação em cursos do Programa Mulheres Mil não interferiu significativamente na Insegurança Alimentar das estudantes $(\mathrm{p}=0,291)$, embora o percentual de inseguras tenha aumentado de $77,1 \%(\mathrm{n}=296)$ para $80,2 \%$ $(\mathrm{n}=308)$ entre o início e ao menos dois meses após haverem terminado seus cursos.

Em relação à escolaridade e à situação de Insegurança Alimentar das participantes, os resultados revelam que há uma diferença significativa, tanto para o momento inicial de aplicação quanto para o momento final da aplicação do instrumento (Tabela 2).

Os perfis de Insegurança Alimentar nos diferentes tempos de aplicação do questionário também, indicaram que a Renda Mensal Familiar apresentou diferença estatística significativa entre os dois momentos estudados, ou seja, ao início do curso e pelo menos dois meses após a conclusão. Cabe ressaltar que embora a renda tenha aumentado, ainda assim não foi suficiente para reduzir significativamente a Insegurança Alimentar das participantes e seus domićlios (Tabela 3).

A partir da observação do percentual de egressas que são as principais referências de renda de seus domicílios, constatou-se que no grupo de Insegurança Alimentar o percentual foi de $47,4 \%$, enquanto que no grupo Com Segurança Alimentar foi de $34,9 \%$. Quanto ao Número de Dependentes da Renda Mensal Familiar, a maioria das respondentes nos dois grupos declarou ser de até quatro pessoas, entretanto, ao analisarmos a relação percentual entre os grupos de domicílios seguros e inseguros, percebe-se que nos inseguros o percentual das que declararam Mais de Quatro Dependentes da Renda Mensal Domiciliar foi de $35,5 \%$, ou seja, mais que duas vezes o percentual entre as mulheres cujos domicílios estavam seguros $(17,4 \%)$. 
Tabela 1. Perfil Demográfico e Socioeconômico e Medida de Associação por Tempo de Aplicação dos Questionários das Alunas do Programa Mulheres Mil.

\begin{tabular}{|c|c|c|c|c|c|c|}
\hline & & \multicolumn{5}{|c|}{ Tempo de aplicação do questionário } \\
\hline \multirow{2}{*}{\multicolumn{2}{|c|}{ Variável }} & \multicolumn{2}{|c|}{ Início do Curso } & \multicolumn{3}{|c|}{ Após Conclusão do Curso } \\
\hline & & $\mathbf{N}$ & $\%$ & $\mathbf{N}$ & $\%$ & $\mathbf{p}$ \\
\hline \multirow[t]{2}{*}{ Faixa Etária ${ }^{* *}$} & $<40$ anos & 191 & 50,8 & - & - & - \\
\hline & $>40$ anos & 185 & 49,2 & - & - & \\
\hline \multirow[t]{2}{*}{ Estado Civil ${ }^{\star *}$} & Com companheiro & 163 & 43,0 & - & - & - \\
\hline & Sem companheiro & 216 & 57,0 & - & - & \\
\hline \multirow[t]{2}{*}{ Escolaridade $^{* *}$} & $\begin{array}{l}\text { Até o ensino } \\
\text { fundamental II completo }\end{array}$ & 191 & 51,1 & - & - & - \\
\hline & $\begin{array}{l}\text { Ensino médio } \\
\text { incompleto ou mais }\end{array}$ & 183 & 48,9 & - & - & - \\
\hline \multirow{2}{*}{$\begin{array}{l}\text { Respondente como Principal } \\
\text { Fonte de Renda do Domicílio }\end{array}$} & Sim & 168 & 44,6 & 158 & 42,4 & 0,543 \\
\hline & Não & 209 & 55,4 & 215 & 57,6 & \\
\hline \multirow[t]{2}{*}{ Renda Mensal Familiar } & Até $1 \mathrm{SM}$ & 275 & 73,9 & 183 & 48,5 & $0,001^{\star}$ \\
\hline & $>$ que $1 \mathrm{SM}$ & 97 & 26,1 & 194 & 51,5 & \\
\hline \multirow{2}{*}{$\begin{array}{l}\text { Número de Pessoas no } \\
\text { Domicílio }\end{array}$} & Até 4 pessoas & 260 & 68,6 & 285 & 74,4 & $0,045^{\star}$ \\
\hline & $>4$ pessoas & 119 & 31,4 & 98 & 25,6 & \\
\hline \multirow[t]{2}{*}{ Gasto com Alimentação } & Até 1 SM & 322 & 89,2 & 312 & 85,5 & 0,132 \\
\hline & $>1 \mathrm{SM}$ & 39 & 10,8 & 53 & 14,5 & \\
\hline \multirow[t]{2}{*}{ Zona da Moradia } & Zona Rural & 78 & 20,7 & 79 & 21,0 & 0,943 \\
\hline & Zona Urbana & 298 & 79,3 & 298 & 79,0 & \\
\hline
\end{tabular}

${ }^{*} \mathrm{p}<0,05$; ${ }^{* *}$ Essas informações foram coletadas apenas no momento inicial do curso.

Tabela 2. Perfil da Escolaridade e Insegurança Alimentar por Tempo de Aplicação do Questionário das Alunas do Programa Mulheres Mil.

\begin{tabular}{|c|c|c|c|c|c|c|c|c|c|c|c|}
\hline \multirow{4}{*}{\multicolumn{2}{|c|}{ Variável }} & \multicolumn{10}{|c|}{ Tempo de aplicação do questionário } \\
\hline & & \multicolumn{5}{|c|}{ Início do Curso } & \multicolumn{5}{|c|}{ Após a Conclusão do Curso } \\
\hline & & \multicolumn{2}{|c|}{$\begin{array}{c}\text { Até o Ensino } \\
\text { Fundamental II } \\
\text { Completo }\end{array}$} & \multicolumn{3}{|c|}{$\begin{array}{c}\text { Ensino Médio } \\
\text { Incompleto ou mais }\end{array}$} & \multicolumn{2}{|c|}{$\begin{array}{l}\text { Até o Ensino } \\
\text { Fundamental II } \\
\text { Completo }\end{array}$} & \multicolumn{3}{|c|}{$\begin{array}{c}\text { Ensino Médio } \\
\text { Incompleto ou mais }\end{array}$} \\
\hline & & $\mathbf{N}$ & $\%$ & $\mathbf{N}$ & $\%$ & $\mathbf{p}$ & $\mathbf{N}$ & $\%$ & $\mathbf{N}$ & $\%$ & $\mathbf{p}$ \\
\hline Insegurança & S A & 34 & 17,8 & 51 & 27,9 & $0,026^{*}$ & 29 & 15,2 & 44 & 24,0 & $0,037^{\star}$ \\
\hline Alimentar & I A & 157 & 82,2 & 132 & 72,1 & & 162 & 84,8 & 139 & 76,0 & \\
\hline
\end{tabular}

* Legenda: S A - Segurança Alimentar e I A - Insegurança Alimentar.

Tabela 3. Perfil de Renda e Insegurança Alimentar por Tempo de Aplicação do Questionário das Alunas do Programa Mulheres Mil.

\begin{tabular}{|c|c|c|c|c|c|c|c|c|c|c|c|}
\hline \multirow{4}{*}{\multicolumn{2}{|c|}{ Variável }} & \multicolumn{9}{|c|}{ Estado de Insegurança Alimentar } & \\
\hline & & \multicolumn{4}{|c|}{ Segurança Alimentar } & \multicolumn{6}{|c|}{ Insegurança Alimentar } \\
\hline & & \multicolumn{2}{|c|}{$\begin{array}{l}\text { Início do } \\
\text { curso }\end{array}$} & \multicolumn{2}{|c|}{$\begin{array}{c}\text { Após a } \\
\text { Conclusão do } \\
\text { Curso }\end{array}$} & \multicolumn{3}{|c|}{$\begin{array}{l}\text { Início do } \\
\text { curso }\end{array}$} & \multicolumn{2}{|c|}{$\begin{array}{c}\text { Após a } \\
\text { Conclusão do } \\
\text { Curso }\end{array}$} & \multirow[b]{2}{*}{$\mathbf{p}$} \\
\hline & & $\mathbf{N}$ & $\%$ & $\mathbf{N}$ & $\%$ & $\mathbf{p}$ & $\mathbf{N}$ & $\%$ & $\mathbf{N}$ & $\%$ & \\
\hline Renda Mensal & Até $1 \mathrm{SM}$ & 46 & 54,8 & 20 & 26,7 & $0,000^{*}$ & 229 & 79,5 & 163 & 54,0 & $0,000^{*}$ \\
\hline Familiar & $>1 \mathrm{SM}$ & 38 & 45,2 & 55 & 73,3 & & 59 & 20,5 & 139 & 46,0 & \\
\hline
\end{tabular}

SM = salário mínimo. No período de aplicação do questionário o salário mínimo equivalia a R\$ 678,00. 


\section{Discussão}

Variáveis Demográficas e Socioeconômicas

A maioria das mulheres tinham menos que 40 anos, sem companheiro, haviam cursado até o Ensino fundamental II completo, não eram a principal fonte de Renda do Domicílio, a Renda Mensal Familiar era de Até 1 Salário Mínimo, seu Gasto com Alimentação era também de 1 Salário Mínimo, e seus domicílios localizava-se na Zona Urbana. Tais resultados são semelhantes aos dados de outros estudos no Brasil ${ }^{2,15}$, e aos dados do Instituto Brasileiro de Geografia e Estatística IBGE $^{19}$ para populações de programas que atendem mulheres em vulnerabilidade social.

Considerando ainda os resultados relacionados à renda dos domicílios das participantes, e a presença de até quatro dependentes da renda mensal familiar, infere-se que a maioria das participantes tem renda per capita de "até $1 / 4$ do salário mínimo", evidenciando o estado de vulnerabilidade social dessas mulheres. Outro aspecto é a relação entre famílias chefiadas por mulheres, classe de rendimento e a prevalência de IA, como já apontado por Brito e Costa em $2015^{2}$ e pelo IBGE no ano anterior ${ }^{8}$.

$\mathrm{Na}$ mesma direção, Marin-Leon et al. ${ }^{20}$ encontraram nos domicílios com renda per capita de até um salário mínimo uma maior prevalência de insegurança alimentar e a presença de outras características como: localização em áreas metropolitanas, pessoa de referência do sexo feminino de cor negra com tempo de estudo inferior a cinco anos e mais de seis moradores por domicílio. Tais achados evidenciam que os domicílios chefiados por mulheres de baixa escolaridade, principalmente se forem negras, estão muito mais sujeitos a insegurança alimentar.

A associação entre a Insegurança Alimentar e a desnutrição já foi amplamente estudada em passado recente. Entretanto estudos mais atuais vêm apontando o efeito da insegurança Alimentar sobra à obesidade infantil ${ }^{21}$ e associada à pior qualidade da alimentação com redução da ingestão de frutas e hortaliças e ao aumento do consumo de carboidratos refinados, gorduras, deficiência de micronutrientes, com pior estado de saúde e situações de estresse, inclusive com estudos mundiais ${ }^{22}$ e revisão sistemática ${ }^{23}$. Portanto, as mulheres do presente estudo e os residentes dos seus domicílios estão sujeitos a riscos para além da falta de alimento.

Observa-se no caso da utilização intrafamiliar de recursos provenientes de programas de trans- ferência de renda, que esta dependerá de quem os controla no âmbito da família, e das diferentes necessidades vivenciadas. Nas situações nas quais a mulher é a receptora preferencial dos recursos, pressupõe-se que há um maior direcionamento dos gastos com a alimentação e com as crianças. Entretanto, deve-se considerar que esta dinâmica pode variar de acordo com as relações de gênero que se estabelecem em cada contexto familiar ${ }^{24}$.

Destaca-se que a não alteração significativa da situação de Insegurança Alimentar, mesmo diante do aumento na renda das participantes, também já foi verificada em estudos que avaliam programas voltados apenas para distribuição de renda. Possivelmente neste estudo tal evidência se deva ao pouco tempo entre a primeira e a segunda aplicação da Escala Brasileira de Insegurança alimentar (seis meses), ou ainda a percepção desta amostra de que não tenham meios para adquirir alimentos em quantidade suficiente podendo assim se considerar vítimas da fome ainda que não tenham sinais reconhecíveis ${ }^{25}$. Além disso, mesmo não passando fome, algumas pessoas podem sentir um medo justificável de privações futuras como identificado pela FAO já em $2002^{26}$.

Por isso, avaliar a percepção da fome e o comportamento a ela correlato, muito mais que uma aproximação ou uma medida indireta; é uma tentativa de chegar ao coração do problema da fome.

Diversos autores ${ }^{2,3}$ destacam a relevância de ações voltadas para a condição feminina nos aspectos educacionais e profissionais, entre outros.

A associação existente entre SAN e educação é posta em evidência por meio de vários estudos, como exemplo, a análise realizada pelo Núcleo de Pesquisas Epidemiológicas em Nutrição e Saúde da Universidade de São Paulo. Tal pesquisa, realizada no Nordeste do Brasil, teve por objetivo descobrir as causas do declínio acelerado da desnutrição nas crianças com cinco anos ou menos entre 1986 e 2006. Os resultados mostraram que melhorias na escolaridade materna eram um dos principais fatores que contribuíram para esta mudança ${ }^{27}$.

Outro resultado que ratifica a vulnerabilidade social das entrevistadas é o percentual de domicílios chefiados por mulheres. De acordo com a $\mathrm{PNAD}^{8}$, os domicílios cujo sexo da pessoa de referência é feminino apresentam 9,3\% de prevalência de insegurança alimentar moderada ou grave, já no caso masculino esse percentual cai para $6,9 \%$. Note que esses resultados não se confirmam no estudo de Godoy et al. ${ }^{17}$, possivelmente porque o Programa Restaurantes Populares é um programa aberto à população em geral, não sendo 
necessário um cadastro para consumir em um dos seus restaurantes. As autoras não encontraram diferenças significativas na segurança alimentar dos domicílios quando o sexo era considerado.

Para Siliprandi ${ }^{6}$ é de fácil compreensão a maior vulnerabilidade das mulheres em relação à desnutrição, uma vez que essa situação relacionase diretamente com o acesso aos recursos produtivos, representando uma importante característica das relações de gênero e segurança alimentar. Maluf $^{3}$, Brito e Costa ${ }^{2}$ destacam também que os temas relativos a gênero deverão ser enfrentados, assim como reconhecidas as múltiplas atribuições das mulheres em relação à produção ou obtenção, preparação e a divisão dos alimentos entre seus familiares.

\section{Situação de Insegurança Alimentar}

Apesar de percentuais de Insegurança Alimentar desta pesquisa serem diametralmente opostos aos dados da $\mathrm{PNAD}^{8}$, tal resultado já era esperado. Nossas respondentes foram captadas de um programa de qualificação profissional de mulheres em vulnerabilidade social, o que mostra, também, a sensibilidade do programa para atender a população a que se destina ${ }^{28}$.

Além disso, os cursos eram ofertados em escolas próximas ou dentro dos centros urbanos, o que fez com que a maioria das alunas morasse nessas áreas. Os dados da $\mathrm{PNAD}^{8}$ apontaram que na área urbana a Insegurança Alimentar da população brasileira atingia à época, 20,5\% na área urbana e $35,3 \%$ na área rural. Aqui novamente a forma de coleta dos dados é o influenciador.

Anschau et al. ${ }^{15}$ em estudo com 421 domicílios em Toledo-PR, encontrou maior Insegurança Alimentar entre famílias beneficiárias de Programas de Transferência de Renda, com menos escolaridade e chefiado por mulheres. Os autores destacam como conclusão do estudo a necessidade que desenvolver novas alternativas de renda, têm papel relevante na proteção de tais grupos, e que se espera que tais intervenções tornem as famílias pobres, menos pobres.

Por outro lado Godoy et al. ${ }^{17}$ encontraram percentuais menores de Insegurança Alimentar (40,6\%) em seu estudo com 1.637 participantes do Programa Restaurantes Populares. Tal resultado, mesmo menor, esteve significativamente associado à renda e a escolaridade. A chance de o usuário estar em insegurança alimentar aumenta de forma inversamente proporcional à renda. Segundo as autoras, usuários com renda inferior a 1/4 do salário mínimo têm 3,67 vezes mais chances de estar em insegurança alimentar do que aqueles que recebem mais que dois salários mínimos.

Salles-Costa et al. ${ }^{29}$ em pesquisa em Duque de Caxias-RJ também relataram um percentual de 53,8\% de Insegurança Alimentar relacionado também, entre outros fatores, inversamente a Renda Familiar Mensal e a Escolaridade do chefe da família. Na mesma direção, Facchini et al. ${ }^{30}$, Santos et al. ${ }^{31}$ e Yang ${ }^{32}$ também associaram essas mesmas variáveis à Insegurança Alimentar.

Portanto, a renda e a escolaridade foram novamente confirmadas como muito importantes para a Segurança Alimentar. Todos esses achados corroboram a importância tanto de Programas de Transferência de Renda quanto de programas cujo foco é a população em vulnerabilidade social.

Nesse momento de grave crise que atravessa o país e em que os programas sociais são colocados em xeque é necessário que os recursos tanto materiais quanto humanos sejam investidos em ações tanto que contemplem não só ações de curto prazo, mas também aquelas que deem ferramentas que no médio prazo retire essas famílias da condição de vulnerabilidade ${ }^{33}$.

\section{Conclusões}

Diante dos resultados encontrados, a presente pesquisa concluiu que a participação no programa Mulheres Mil pode contribuir para redução da vulnerabilidade à insegurança alimentar na medida em que proporcione avanços diretamente relacionados aos determinantes da SAN, como caso investigado, à renda. $\mathrm{O}$ fato de o Programa atender mulheres em níveis elevados de vulnerabilidade social, inclusive de Insegurança Alimentar, ratifica a relevância de políticas públicas dessa natureza.

Entretanto, para uma compreensão mais aprofundada dessas relações, sugere-se que mais estudos qualitativos sejam realizados com objetivo de analisar diferentes dimensões de programas e políticas públicas e suas possíveis relações com a SAN, especialmente com recorte de gênero feminino. 


\section{Colaboradores}

JM Campos coordenou o desenho do estudo, realizou toda a coleta de dados in loco, ajudou a redigir o manuscrito e deu a aprovação final da versão a ser publicada. RCCA Akutsu desenho do estudo, realizou a análise estatística e participou da redação do manuscrito e do desenho do estudo. ICR Silva realizou a análise estatística, participou da redação do manuscrito e revisão crítica de importante conteúdo intelectual. KS Oliveira participou da redação do manuscrito e revisão crítica de importante conteúdo intelectual. $\mathrm{R}$ Monteiro participou da redação do manuscrito e do desenho do estudo. Todos os autores leram e aprovaram o manuscrito final.

\section{Referências}

1. Woortmann EF. Da complementaridade à dependência: espaço, tempo e gênero em comunidades "pesqueiras" do Nordeste. Rev Bras Cien Sociais 1992; 7(18):38-52.

2. Brito JG, Costa ER. Titularidade feminina no Programa Bolsa Família: questões de gênero e segurança alimentar. TROPOS 2015; 1(3):1-17.

3. Maluf RSJ. Segurança alimentar e nutricional. Petrópolis: Vozes; 2007.

4. Instituto de Pesquisa Econômica Aplicada (IPEA), ONU Mulheres, Secretaria de Políticas para as Milheres (SPM), Secretaria de Políticas de Promoção da Igualdade Racial (SEPPIR). Retrato das desigualdades de gênero e raça. $4^{\mathrm{a}}$ ed. Brasília: IPEA; 2011.

5. Bellows A, Scherbaum V, Lemke S, Jenderedjian A, Viana RSG. Gender-specific risks and accountability: women, nutrition and the right to food. Right Food Nutr Watch 2011; 1:23-29.

6. Siliprandi E. Políticas de segurança alimentar e relações de gênero. Cad Debate 2004; 11:38-57.

7. Carrasco C. La sostenibilidad de la vida humana:¿un asunto de mujeres? Mientras tanto 2001; 82:43-70.

8. Instituto Brasileiro de Geografia Estatística (IBGE), Pesquisa Nacional por Amostra de Domicílios (PNAD). Segurança Alimentar: 2004/2013: Brasil, grandes regiões e unidades da federação. Rio de Janeiro: IBGE; 2014.

9. Hoffmann R. Brasil, 2013: mais segurança alimentar. Segurança Alimentar Nutricional 2014; 21(2):422-436.

10. Instituto Brasileiro de Geografia Estatística (IBGE), Pesquisa Nacional por Amostra de Domicílios (PNAD). Segurança alimentar 2004/2009: Brasil, grandes regiões e unidades da federação. Rio de Janeiro: IBGE 2010.

11. Barroso C. Metas de desenvolvimento do milênio, educação e igualdade de gênero. Cad Pesquisa 2004; 34(123):573-582.

12. Le Bihan G, Delpeuch F, Maire B. Alimentação, nutrição e políticas públicas. São Paulo: Instituto Pólis; 2003.

13. Ministério do Desenvolvimento Social e Combate à Fome (MDS). O Brasil sem miséria. Brasília: MDS; 2014.

14. Brasil. Ministério da Educação. Portaria $\mathrm{n}^{\mathrm{o}} 1.1015$, de 21 de julho de 2011. Institui o Programa Nacional Mulheres. Diário Oficial da União; 2011.

15. Anschau FR, Matsuo T, Segall-Correa AM. Food Insecurity Among Recipients Of Government Assistance [Insegurança Alimentar Entre Beneficiários De Programas De Transferência De Renda]. Rev Nutr 2012; 25(2):177-189.

16. Panigassi G, Segall-Corrêa AM, Marin-León L, Pérez-Escamilla R, Sampaio MFA, Maranha LK. Insegurança alimentar como indicador de iniqüidade: análise de inquérito populacional. Cad Saude Publica 2008; 24(10):2376-2384. 
17. Godoy K, Sávio KEO, Akutsu RC, Gubert MB, Botelho RBA. Food insecurity and nutritional status of individuals in a socially vulnerable situation in Brazil. Cien Saude Colet 2017; 22(2):607-616.

18. Carter KN, Kruse K, Blakely T, Collings S. The association of food security with psychological distress in New Zealand and any gender differences. Soc Sci Med 2011; 72(9):1463-1471.

19. Instituto Brasileiro de Geografia e Estatstica (IBGE). Pesquisa Nacional por Amostras de Domicílios Contínua (PNAD Contínua). Rio de Janeiro: IBGE; 2018.

20. Marin-Leon L, Francisco PMSB, Segall-Corrêa AM, Panigassi G. Bens de consumo e insegurança alimentar: diferenças de gênero, cor de pele autorreferida e condição socioeconômica. Rev Bras Epidemiol 2011; 14(3):398-410.

21. Kaur J, Lamb MM, Ogden CL. The association between food insecurity and obesity in children--The National Health and Nutrition Examination Survey. $J$ Academy Nutr Dietetics 2015; 115(5):751-758.

22. Food and Agriculture Organization of the United Nations (FAO), International Fund for Agricultural Development (IFAD), World Food Programme (WFP). The state of food insecurity in the world 2014. Strengthening the enabling environment for food security and nutrition. Rome: FAO; 2014

23. Rocha NP, Milagres LC, Novaes JF, Franceschini SCC. Associação de insegurança alimentar e nutricional com fatores de risco cardiometabólicos na infância e adolescência: uma revisão sistemática. Rev Paulista Pediatr 2016; 34(2):225-233.

24. Leão MM, Recine E. O direito humano à alimentação adequada. Nutrição em saúde pública. Rio de Janeiro: Rubio; 2011.

25. Pessanha L, Vannier-Santos C, Mitchell PV. Indicadores para avaliar a Segurança Alimentar e Nutricional e a garantia do Direito Humano à Alimentação: metodologias e fontes de dados. Anais 2016: 1:1-21.

26. Food and Agriculture Organization of the United Nations (FAO). Food Insecurity Vulnerability Information and Mapping Systems (FIVIMS) [Internet]. Rome: FAO; 2009. Disponível em: http://www.fao.org/3/ AB990E/ab990e05.htm

27. Lima ALL, Silva ACF, Konno SC, Conde WL, Benicio MHD'A, Monteiro CA. Causas de la disminución acelerada de la desnutrición infantil en Noreste de Brasil (1986-1996-2006). Rev Saude Publica 2010; 44(1):17-27.

28. Alaniz EP, Mantovani TRDA, Luvizotto CK. Política de formação profissional no Brasil: uma análise do Programa Mulheres Mil. Org Demo 2016; 17(1):85100.

29. Salles-Costa R, Pereira RA, Vasconcellos MTL, Veiga GV, Marins VMR, Jardim BC, Gomes FS, Sichieri R. Associação entre fatores socioeconômicos e insegurança alimentar: estudo de base populacional na Região Metropolitana do Rio de Janeiro, Brasil. Revi Nutr 2008; 21:99s-109s.
30. Facchini LA, Nunes BP, Motta JVS, Tomasi E, Silva SM, Thumé E, Silveira DS, Siqueira FV, Dilélio AS, Saes MO, Miranda VIA, Volz PM, Osório A, Fassa AG. Insegurança alimentar no Nordeste e Sul do Brasil: magnitude, fatores associados e padrões de renda per capita para redução das iniquidades. Cad Saude Publica 2014; 30(1):161-174.

31. Santos JV, Gigante DP, Domingues MR. Prevalência de insegurança alimentar em Pelotas, Rio Grande do Sul, Brasil, e estado nutricional de indivíduos que vivem nessa condição. Cad Saude Publica 2010; 26(1):41-49.

32. Yang YJ. Socio-demographic characteristics, nutrient intakes and mental health status of older Korean adults depending on household food security: based on the 2008-2010 Korea National Health and Nutrition Examination Survey. Korean J Comm Nutr 2015; 20(1):30-40.

33. Santarelli M, Burity V, Silva LNB, Prates L, Rizzolo A, Rocha NC, Trabuco L. Da democratização ao golpe: avanços e retrocessos na garantia do direito humano à alimentação e à nutrição adequadas no Brasil. Brasília: FIAN Brasil; 2017.

Artigo apresentado em 04/12/2017

Aprovado em 08/08/2018

Versão final apresentada em 10/08/2018 
